\title{
On the role of figural organization in perceptual transparency
}

\author{
JACOB BECK and RICHARD IVRY \\ University of Oregon, Eugene, Oregon
}

\begin{abstract}
Metelli (1974) made an important contribution by identifying order and magnitude restrictions for a pattern of intensities and showing that when they are satisfied the perception of transparency readily occurs. These restrictions were derived from a physical model of transparency. We argue that the visual system does not use intensity information to compute indices of transmittance and reflectance analogous to what an optical engineer might do in describing a physical instance of transparency. Rather, a lightness pattern affects perceptual transparency, just as geometric properties do, through processes that impose an organization on sensory information rather than through processes that recover quantitative descriptions. In the absence of depth cues, such as stereopsis and motion parallax, the perception of transparency occurs when the lightness relations in a pattern favor the perception of a continuous boundary across $x$-junctions. We present evidence for two kinds of violations of the order and magnitude restrictions: simple and strong. Transparency judgments, although reduced in number, still occur for simple violations of the order and the magnitude restrictions. Transparency judgments occur relatively infrequently for strong violations. A physical model of transparency fails to capture the difference between simple and strong violations of the order and magnitude restrictions. We discuss (1) the basis for differentiating between simple and strong violations of the order and magnitude restrictions, (2) the effect simple and strong violations have on the perception of transparency, and (3) the occurrence of transparency with and without color constancy (i.e., the color seen through the transparent surface looks or fails to look the same as the color seen directly).
\end{abstract}

Metelli (1974) proposed a model for the perception of transparency based on a physical model of transparency. Beck, Prazdny, and Ivry (1984) reported findings that challenge the adequacy of the Metelli model. The implications of these findings were not made fully clear in the earlier paper; thus, in this paper, we present the Metelli model, the difficulties we see with the Metelli model, an alternative model based on processes of figural organization, and an experiment that further clarifies the conditions for the perception of transparency.

\section{Episcotister Model}

Metelli (1974) proposed a model for the intensity relationships in perceptual transparency based upon transparency produced by a rotating episcotister (a disk with a sector cut out). Rotating an episcotister in front of surfaces $A$ and $B$ (Figure 1) produces the perception of a transparent color (regions $d$ and $c$ ) lying in front of surfaces $A$ and $B$. The apparent reflectances of regions $d$ and $c$ are determined as follows:

$$
d=\alpha a+(1-\alpha) e
$$

and

$$
c=\alpha b+(1-\alpha) e
$$

This research was supported by National Science Foundation Grant INT-8418187 and by Air Force of Scientific Research Grant AFOSR85-0359. Requests for reprints should be sent to Jacob Beck, Department of Psychology, University of Oregon, Eugene, OR 97403. where $\alpha$ is the proportion of light reflected from surface $A$ (corresponding to the areal fraction occupied by the open sector of the episcotister), $1-\alpha$ is the proportion of light reflected from the blades of the episcotister (corresponding to the areal fraction occupied by the blades of the episcotister), $a$ is the reflectance of surface $A, b$ is the reflectance of surface $\mathrm{B}$, and $e$ is the reflectance of the episcotister blades. Solving Equations 1 and 2 for $\alpha$ and $e$ yields

$$
\alpha=(d-c) /(a-b)
$$

and

$$
e=(a c-b d) /(a+c)-(b+d) .
$$

Since $\alpha$ is restricted to values between 0 and 1 , Equation 3 implies that (i) If $a>b$, then $d>c$, and if $a<b$, then $d<c$; (ii) The absolute difference $|a-b|$ must be greater than the absolute difference $|d-c|$. Since $e$, the reflectance of the episcotister blades, is also restricted to values equal to or between 0 and 1, Equation 4 implies that (i*) If $(a+c)>(b+d)$, then $a c>b d$, and if $(a+c)$ $<(b+d)$, then $a c<b d$. (ii*) The absolute difference $|(a+c)-(b+d)|$ must be equal to or greater than the absolute difference $|a c-b d|$.

Restrictions $i$ and $i^{*}$ are restrictions on the order of the intensities and ensure that $\alpha$ and $e$ are positive. Restrictions ii and ii* are restrictions on the magnitudes of the intensities and ensure that $\alpha$ and $e$ are less than 1. Beck et al. (1984) showed that restrictions $\mathrm{i}, \mathrm{ii}, \mathrm{i}^{*}$, and $\mathrm{ii}^{*}$ hold 
A

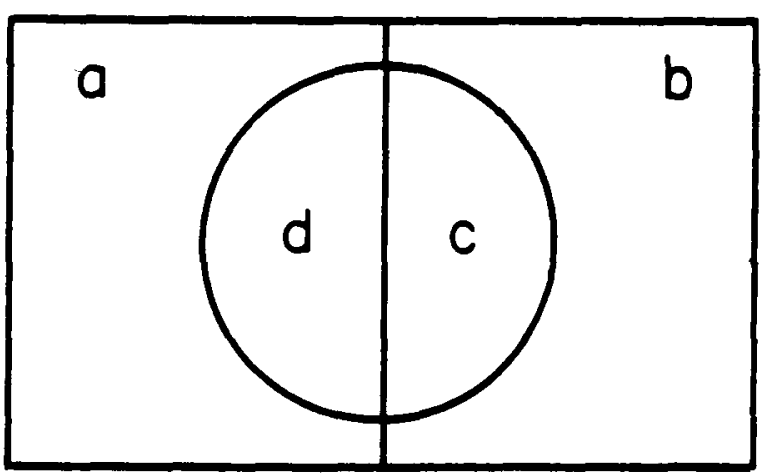

Figure 1. The proximal stimulus resulting from an episcotister's rotating in front of two surfaces that differ in reflectance. Capital letters $A$ and $B$ indicate the background surfaces. Lowercase letters indicate regions of differing reflectance.

for transparency produced by a filter, as well as for transparency produced by an episcotister ${ }^{1}$; that is, if these restrictions are violated, transparency is physically impossible under the assumptions that the transparent surface has a uniform density and a uniform reflectance. The restrictions, therefore, are ecologically valid.

Violations of restrictions $\mathrm{i}^{*}$ and ii* do not adversely affect the perception of transparency (Beck et al., 1984). This is not surprising since Equation 4 is not scale invariant, and actual reflectances are necessary to determine whether the equation is satisfied (Brill, 1986). In contrast, violations of restrictions $i$ and ii markedly reduce the perception of transparency (Beck et al., 1984; Metelli, Da Pos, \& Cavedon, 1985). Violation of only restriction i will be referred to as a simple violation of the order restriction, and violation of only restriction ii as a simple violation of the magnitude restriction.

\section{Difficulties with the Episcotister Model}

Metelli (1974) made an important contribution in identifying the simple order and magnitude restrictions and in showing that when they are satisfied transparency occurs with high frequency. Beck et al. (1984), however, reported four findings that require a reevaluation of the Metelli model.

First, Beck et al. (1984) questioned the interpretation of $\alpha$ as an index of perceived transparency. They showed that the relevant variable for the perception of transparency is lightness, ${ }^{2}$ and not the physical variables of luminance or reflectance. Lightness to a first approximation is a negatively accelerated monotonic function of reflectance (Judd \& Wyszecki, 1963). Since it is monotonic, if restriction i is satisfied in terms of lightness, it is also satisfied in terms of reflectance. However, restriction ii can be satisfied in terms of reflectance values but not in terms of lightness values, and vice versa. Metelli (1985) accepted Beck et al.'s view that lightness is the relevant variable and substituted lightness values in Equation 3 to calculate a perceived transparency index:

$$
\alpha^{\prime}=\left(d^{\prime}-c^{\prime}\right) /\left(a^{\prime}-b^{\prime}\right) \text {. }
$$

Since lightness is a nonlinear function of intensity, $\alpha^{\prime}$ is not a physically correct index of transparency.

Second, Beck et al. (1984) showed that $\alpha^{\prime}$ fails to predict the perceived transparency accurately in many instances. As just indicated, restrictions $\mathrm{i}^{*}$ and ii* can be violated and transparency is readily still seen. In actual instances of transparency, if the reflectance difference $|d-c|$ approaches the reflectance difference $|a-b|$, then the reflectance of region $d$ approaches the reflectance of region $a$, and the reflectance of region $c$ approaches the reflectance of region b (Figure 1). However, if either restrictions $\mathrm{i}^{*}$ or $\mathrm{ii}^{*}$ are violated, it is possible for the lightness difference $|d-c|$ to approach the lightness difference $|a-b|$, and the lightness values of regions $d$ and $c$ would not approach the lightness values of regions a and b. In Experiment 4 of Beck et al. (1984), substituting lightness values in Equation 5 for stimulus 9 predicted a transparency of .96 when the bottom rectangle was seen as transparent and overlying the top square and the background. A transparency of .96 implies that the lightness values of regions $d$ and a should be similar and that the lightness values of regions $c$ and $b$ should be similar. This was clearly not the case. The mean lightness match of region a was a Munsell value (MV) of 7.80; of region $b$, a MV of 6.50; of region c, a MV of 2.65; and of region d, a MV of 3.70. This occurred because the stimulus violated restriction $i^{*}$. The mean of subject's transparency estimates was .38 . Thus, Equation 5 may fail to predict perceived transparency when restrictions $i^{*}$ or ii* are violated. These, of course, are instances of perceived transparency in which transparency is physically impossible.

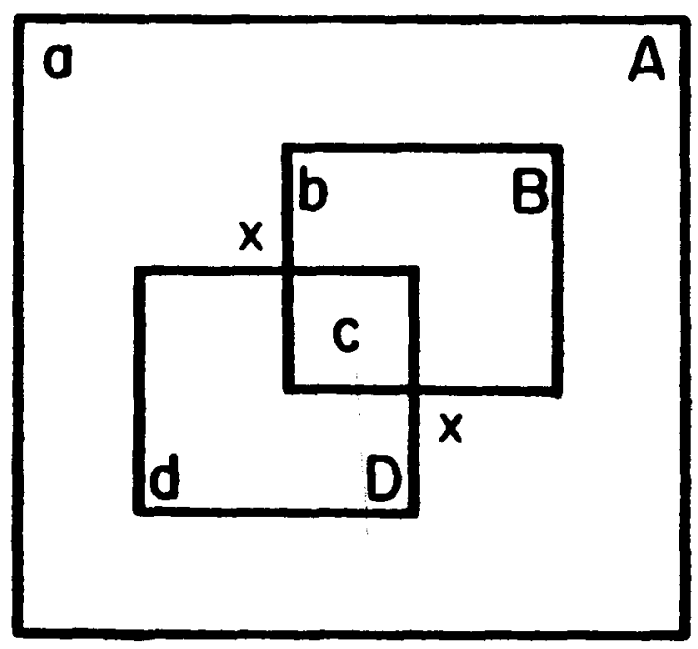

Figure 2. Capital letters indicate the surfaces depicted. Lowercase letters indicate regions of differing lightness. $A$ and $B$ are opaque, and $D$ is a transparent surface. The xs mark x-junctions. 
Third, simple violations of the order and the magnitude restrictions fail to adequately characterize when the perception of transparency occurs. Beck et al. (1984) found that the perception of transparency occurred readily when restrictions $i$ and ii were satisfied. When they were violated, however, stimuli fell into two subgroups. For one subgroup, the perception of transparency still occurred significantly; for the other subgroup, which were termed "strong violations," the perception of transparency was rarely reported.

Figure 2 shows the arrangement of the stimuli in Beck et al. (1984). Capital letters identify depicted surfaces, and lowercase letters identify regions of differing reflec-

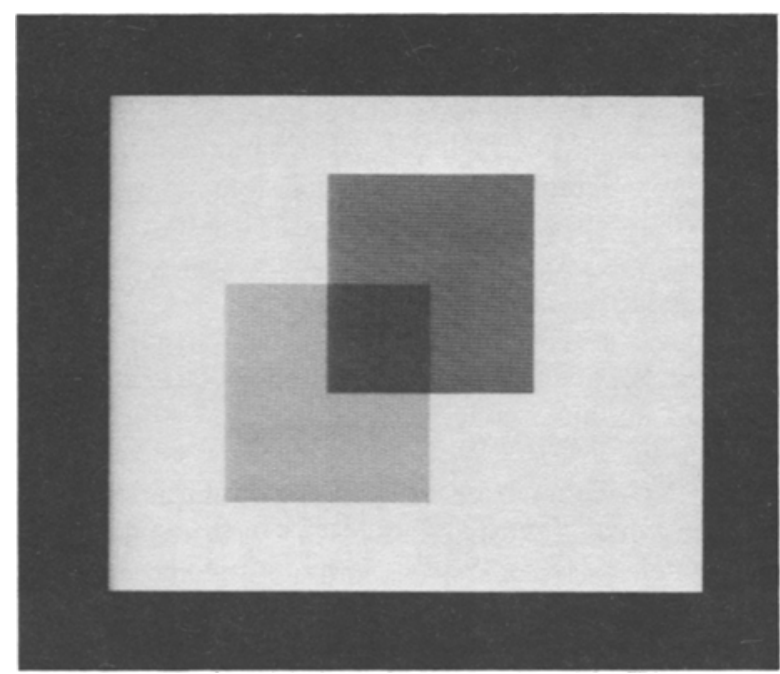

a

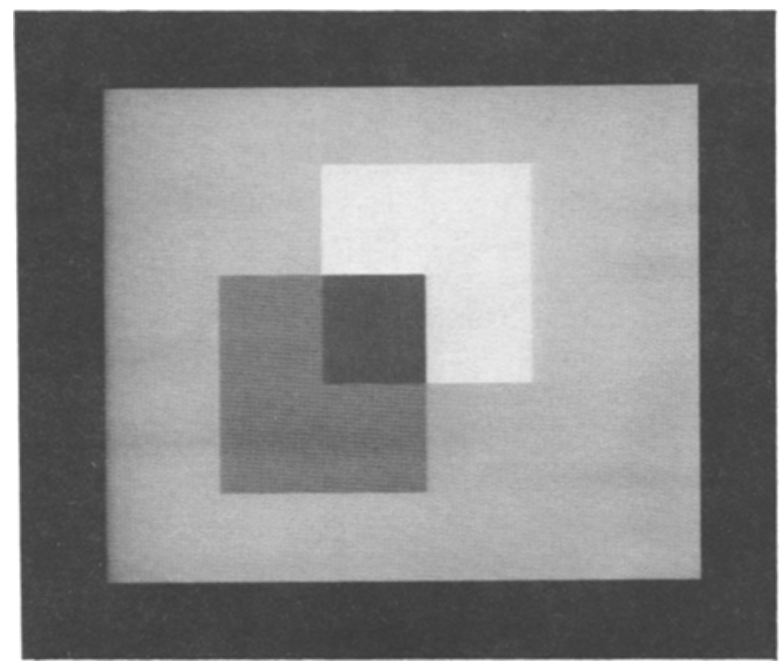

b tance. Our discussion of Figures 2, 3, and 4 assumes that $\mathrm{D}$ is the transparent surface overlying the opaque surfaces A and B. Stimuli $c a d b$ and $c d a b$ both violate restriction i (the order of letters indicate increasing lightness values from lowest to highest). Beck et al. found that no subjects saw cadb (Figure 3d) as transparent, but that 13 of 21 subjects saw cdab (Figure 3b) as transparent. Beck et al. characterized the two types of violations geometrically. Stimulus $c a d b$, but not stimulus $c d a b$, is an example of what they called a crisscross stimulus. In a crisscross stimulus, if the lightness values of the four quadrants at the $x$-junctions (marked by $x s$ in Figure 2) are traced out in increasing magnitude, the lightness values criss-

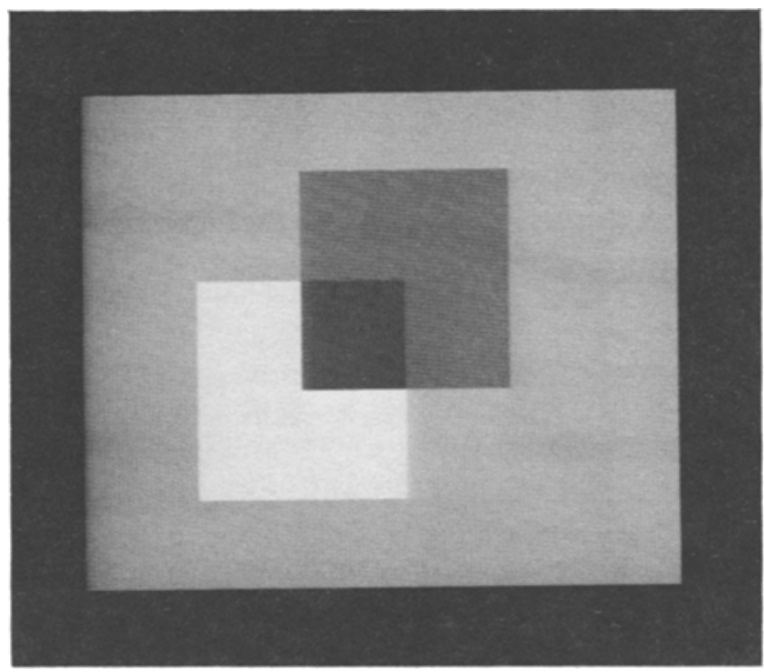

C

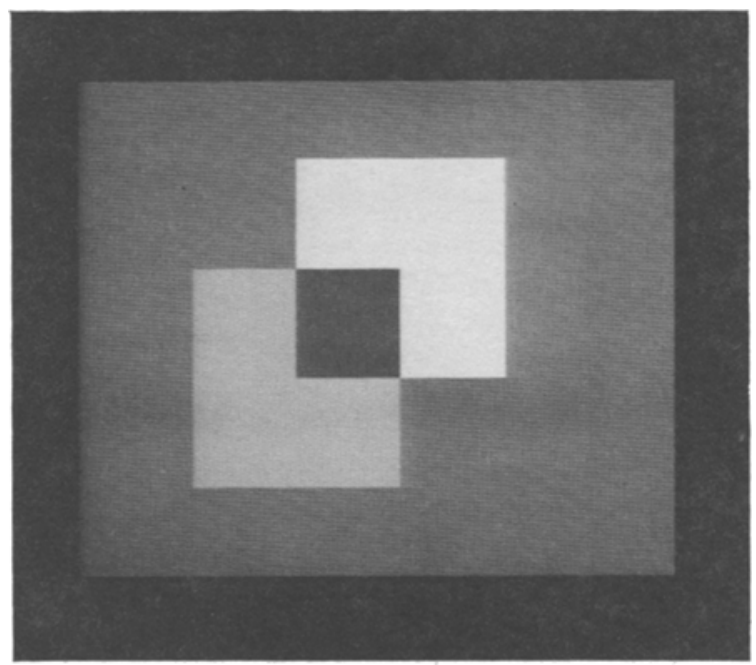

d

Figure 3. Sanple stimuli: (a) Stimulus cbda satisfies restrictions i through iv. (b) Stimulus cdab is a simple violation of the order restriction. It violates only restriction $\mathbf{i}$. (c) Stimulus cbad is a strong violation of the magnitude restriction. It violates restriction iv. (d) Stimulus cadb is a strong violation of the order restriction. It violates restrictions $i$ and iii. 
cross (Figure 4d). A crisscross stimulus represents a strong violation of restriction i. Figure $3 \mathrm{~b}$ shows a simple violation of restriction $i$. When the lightness values of the quadrants at the upper $x$-junction are traced out in increasing magnitude in a simple violation of restriction $i$, the resulting figure is a $C$ or a backward $C$ (Figure $4 b$ ).

Restriction i ensures that the change in the direction of lightness across the vertical boundary of the upper $x$ junction in Figure 2 (the border between the opaque surfaces $A$ and $B$ ) is in the same direction. The corresponding restriction on the direction of lightness changes across the horizontal boundary of the upper $\mathrm{x}$-junction (the border between the transparent surface $D$ and the opaque surfaces $\mathrm{A}$ and $\mathrm{B}$ ) implies that (iii) If $a>d$, then $b>c$, and if $a<d$, then $b<c$. Unlike restriction i, restriction iii does not follow from a physical model of transparency. Restriction $i$ reflects the physical fact that a homogeneous transparent surface cannot invert the order of intensities across a surface boundary; however, a transparent surface can invert the order of intensities across its own boundary. A strong violation of the order restriction violates both restrictions $\mathrm{i}$ and iii. A simple violation of the order restriction violates only restriction i. A strong violation of the order restriction implies a simple violation of the order restriction, but not the reverse.

Two subgroups also were found for violations of the magnitude restriction. Stimuli 13 through 16 in Set 2 of Beck et al. (1984) (the stimulus order was $c d b a$ ) satisfied restriction i but violated restriction ii. The reflectance (and lightness) differences between regions $d$ and $c$ were
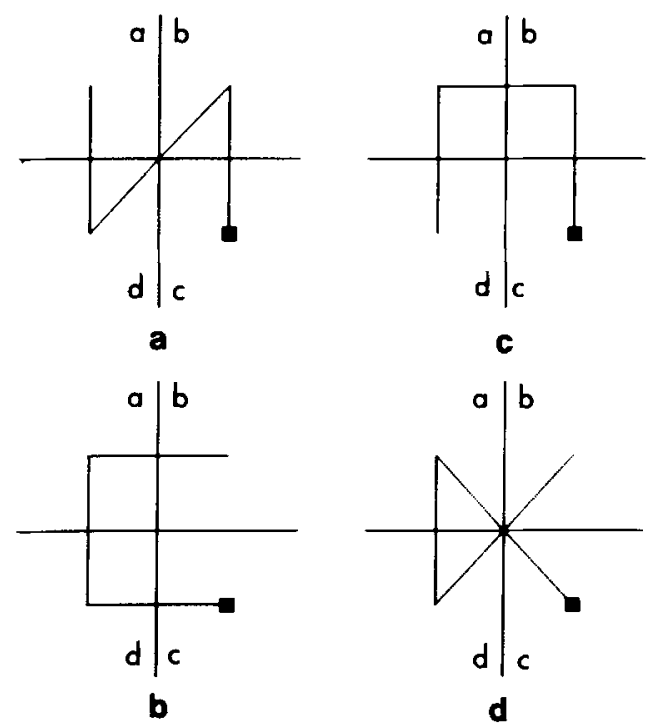

Figure 4. Lightness changes gaross the upper $x$-junction in Figure 2: (a) Stimulus cbda. Stimfili satisfying restrictions i through iv describe a forward or backward $N$ or $Z$. (b) Stimulus cdab. Stimuli violating restriction i describe a forward or backward $C$. (c) Stimulus cbad. Stimuli violating only restriction iv describe an upside-down $U$. d. Stimulus cadb. Stimuli violating restrictions i and iii describe a crisscross figure. greater than those between regions a and $b$. The mean number of transparency judgments for 21 subjects was 12.5. The frequency of transparency judgments varied roughly inversely with the magnitude of the violation of restriction ii for stimulus $c d b a$. Stimulus $c b a d$ also violates restriction ii and satisfies restriction i (Figures 3c and 4c). However, no subjects saw cbad as transparent. Stimulus $c b a d$ violates the stronger restriction: (iv) The lightness interval $[a, b] \in$ the lightness interval $[c, d]$. A strong violation of the magnitude restriction occurs when the lightnesses of the opaque surfaces $A$ and $B$ are contained within the lightness interval $[c, d]$ defined by the transparent regions $c$ and $d$. Simple violations of the magnitude restriction occur when the lightness interval $[c, d]$ is greater than $[a, b]$ but the interval $[a, b]$ is not included within the interval $[c, d]$. Since restriction iv implies restriction ii, a strong magnitude violation implies a simple magnitude violation.

Thus, violations of the order and magnitude restrictions fall into two subgroups: simple violations in which the number of transparency judgments is less but still occurred, and strong violations in which very few transparency judgments occurred. Restrictions $i$ and ii, derived from a physical model of transparency, fail to capture the difference between simple and strong violations of the order and the magnitude restrictions.

Fourth, Beck et al. (1984) argued that it was necessary to clarify the relation between perceptual transparency and color constancy. Transparency occurs in which the color seen through the transparent surface is seen to be the same as the color that is not obscured by the transparent surface. If the transparent surface was removed, one would be confident that the underlying color would be the same as the color not overlaid by the transparent surface. The perception of transparency in this instance exhibits color constancy. The perception of transparency, however, can occur without color constancy. Metzger (1955), for example, showed that perceptual transparency occurs with rotating intersecting circles for color combinations in which the hue of a surface seen through the transparent layer is strikingly different from the hue of the surface seen directly. Beck et al. (1984) suggested that the perception of transparency with a stationary pattern can also occur without color constancy; that is, the lightness of the underlying surface seen directly and through the transparent layer is not seen as the same.

\section{Figural Organization Model}

A change in point of view is required if the psychological variable of lightness, rather than the physical variables of luminance or reflectance, is the relevant variable (Beck, 1986a, 1986b). In perceiving transparency, the visual system is not solving equations derived from a quantitative model of image photometry. Rather, the perception of transparency depends on processes of figural organization. Figural conditions have long been recognized to affect the perception of transparency. What we are proposing is that the lightness values (or, more gener- 
ally, the color attributes) affect perceptual transparency, just as geometric properties do, through processes that impose figural organization on sensory information rather than through processes that recover quantitative descriptions from sensory information. To render our hypothesis tenable, we need to interpret how simple and strong violations of the order and magnitude restrictions affect figural organization.

Fuchs (1923) showed that the perception of transparency with pictorial stimuli is facilitated when figures overlap. The overlapping of surfaces $B$ and $D$ yield $x$-junctions, as shown in Figure 2. Continuity of the boundary lines at an $\mathrm{x}$-junction is necessary for the stratification of surfaces that occurs in the perception of transparency. If the horizontal and vertical contours bounding regions $b$ and $\mathrm{d}$ do not appear to terminate at the $\mathrm{x}$-junction, but rather are seen as the continuations of the edges of surfaces $B$ and $D$, then region $c$ is not seen as an independent region. Instead, it is seen as belonging to either surface B or surface $D$. We hypothesize that transparency is then seen with either surface $D$ overlapping surface $B$ or vice versa. On the other hand, if the horizontal and vertical lines bounding regions $b$ and $d$ are seen as terminating at the $\mathrm{x}$-junctions, transparency is not seen, and regions $\mathrm{a}, \mathrm{b}$, $c$, and $\mathrm{d}$ are perceived as separate, coplanar, and nontransparent.

Similarity is an important factor in grouping. There are two kinds of similarity that are important in determining whether region $\mathrm{c}$ is joined to region $\mathrm{d}$ or whether it is seen as a separate region: (1) the similarity of the lightness relations, and (2) the relative similarity of the lightnesses. Simple and strong violations of the order restriction involve the similarity of the lightness relations. Our hypothesis is that the joining of regions $c$ and $d$ is facilitated (and thereby the likelihood of perceptual transparency is increased) when the direction of lightness changes across an $\mathrm{x}$-junction is in the same direction.

For simple violations of the order restriction, the direction of lightness changes at an $\mathrm{x}$-junction is the same across the boundary between the transparent surface $D$ and the opaque surfaces $A$ and $B$, but not across the boundary between the opaque surfaces A and B (see Figure 2). Across the boundary between the opaque surfaces, the direction of lightness changes is inverted. For example, across the boundary between the opaque surfaces $A$ and $B$, in stimulus $c d a b$ (Figure $3 b$ ), region $a$ is darker than region $b$, but region $d$ is lighter than region $c$ (violates restriction $i$ ). Across the boundary between the transparent surface, $D$, and the opaque surfaces, $A$ and $B$, region $a$ is lighter than region $d$ and region $b$ is lighter than region $c$ (satisfies restriction iii). In a crisscross stimulus, or a strong violation of the order restriction, the lightness values change in opposite directions both across the boundary between the opaque surfaces and across the boundary between the transparent and opaque surfaces. For example, across the boundary between the opaque surfaces $A$ and $B$, in stimulus $c a d b$ (Figure 3d), region a is darker than region $b$, but region $d$ is lighter than region $c$ (violates restriction $i$ ).
Across the boundary between the transparent surface, D, and the opaque surfaces, $A$ and $B$, region $a$ is darker than region $\mathrm{d}$ but region $\mathrm{b}$ is lighter than region $\mathrm{c}$ (violates restriction iii). Restriction $i$ facilitates linking at an $x$ junction the contours of the overlying transparent surface (regions $\mathrm{d}$ and $\mathrm{c}$ ) across the boundary between the opaque surfaces A and B. Restriction iii facilitates linking at an $x$-junction the contours of the underlying opaque surface (regions $b$ and $c$ ) across the boundary between the transparent surface D and the opaque surface $B$.

Simple and strong violations of the magnitude restriction involve the relative similarity of the lightnesses. Regions $a$ and $b$ are always seen as separate surfaces. If, for example, in stimulus cdba (Figure 3a), the lightness difference between regions $c$ and $d$ is greater than the lightness difference between regions $a$ and $b$, the likelihood is increased of seeing regions $c$ and $d$ as belonging to separate surfaces. This, we hypothesize, is why violation of the simple magnitude restriction ii adversely affects the perception of transparency. A violation of the strong magnitude restriction iv, such as stimulus cbad (Figure 3c), requires linking the two extreme lightness values in a stimulus-the lightness values of regions $c$ and $d-$ for the perception of transparency to occur. Linking of the two extreme lightness values in a stimulus is highly unlikely, and the perception of transparency would occur only rarely.

\section{EXPERIMENT}

The aims of the experiment were (1) to investigate how restrictions i through iv affect perceptual transparency, and (2) to clarify the relation between these restrictions and the perception of transparency with and without color constancy.

\section{Method}

The experimental stimuli were pictures, printed by a Tektronix 4634 hardcopy unit, of computer-generated images arranged as in Figure 2. Each stimulus consisted of four regions differing in lightness and depicted two surfaces, $B$ and $D$, lying on a larger background surface A. Capital letters in Figure 2 depict surfaces, and lowercase letters indicate regions of differing lightness values. The rectangles $B$ and D were $3.2 \times 3.4 \mathrm{~cm}$, and the area of overlap (region c) was $1.6 \times 1.7 \mathrm{~cm}$. The background rectangle $A$, was $9.2 \times 7.7 \mathrm{~cm}$. The four lightnesses differed by approximately equal amounts, 1.75 Munsell steps, with the highest lightness equal to a Munsell value of approximately 9.25 . A stimulus is identified by a sequence of the four letters, $a, b, c$, and $d$. The letter order indicates increasing lightness values from lowest to highest of the four regions in a stimulus. There were 24 stimuli corresponding to the 24 possible permutations of the four different lightness values. The stimulus pairs listed as satisfying restriction ii in Table 2 (except for pairs $5,6,9$, and 12 ) technically violate the restriction since the absolute difference $|a-b|$ is equal to the absolute difference $|d-c|$. Beck et al. (1984), however, found that the perception of transparency is adversely affected only when restriction ii is clearly violated. The stimuli are, therefore, considered to effectively satisfy restriction ii and are listed in Table 2 as satisfying it.

At the beginning of an experimental session, transparency was illustrated by showing a subject a neutral density filter overlying 
a gray square on a lighter gray background. The subject also was shown that the perception of transparency can occur in a picture. Before beginning the experiment, each subject was presented with 6 sample stimuli. The sample stimuli were computer-generated images arranged as in Figure 2 and taken from earlier studies of transparency. They consisted of 4 pictures that in these studies had been consistently judged transparent and 2 pictures that had consistently been judged as not transparent. For 2 of the 4 sample stimuli judged transparent, the bottom surface D was seen as transparent, and for 2 the top surface B was seen as transparent. Each subject was instructed to report whether the bottom surface D on each of the 6 sample stimuli was perceived as transparent. The subject was asked to check carefully that both the background A and the top surface B were seen through $D$. Only then should a stimulus be reported as transparent. If $A$ or $B$, but not both, was seen through $D$, or if a surface other than the indicated surface (e.g., the top surface B) was seen as transparent, the stimulus was to be reported as not transparent. The purpose of including sample stimuli not seen as transparent and in which surface $B$ rather than surface $D$ was seen as transparent was to check that a subject understood the criteria for reporting a surface as transparent. A subject was then presented with the 24 experimental stimuli and asked to report whether surface $D$ appeared transparent. The sample and experimental stimuli were presented in a tachistoscope for 2 sec each.

Following the transparency judgments, the subject was told that the perception of transparency can occur in two ways. Often the color seen through the transparent surface looks the same as the color of the surface seen directly; that is, one would be surprised if the transparent surface was removed and the color seen through it was not the same as the color of the rest of the surface. The subject was shown an actual instance of transparency in which this was the case. A perception of transparency, however, can also occur in which the color seen through the transparent surface does not look like the color of the surface seen directly; that is, one would not be surprised if the transparent surface was removed and the color seen through it was different from the color of the rest of the surface. A subject was shown a neutral density filter overlying gray papers cut and arranged as in Figure 2, in which the gray papers seen through the filter (regions $c$ and d) differed in lightness from the gray papers not seen through the filter (regions $b$ and $a$ ). The sample stimuli reported transparent on the initial presentation were then presented, and the subject was asked to judge whether the lightnesses of the surfaces seen through the transparent surface appeared to be the same as the lightness of the surface seen directly. Following the sample stimuli, the experimental stimuli reported transparent on the initial presentation were presented, and the subject was asked whether the perceived lightnesses of surface $A$ and surface $B$ seen through the transparent surface $D$ were the same as the lightnesses of surfaces $A$ and $B$ seen directly.

The subject was then presented with the sample and experimental stimuli judged not transparent on the initial presentation. The purpose was to determine whether the stimuli reported as not transparent on the initial presentation was because surface B was seen as transparent. The instructions were the same as on the initial presentation, except that a subject was asked whether surface B appeared transparent. As before, the stimuli judged transparent were presented again, and the subject was asked whether the perceived lightnesses of surface $A$ and surface $D$ seen through the transparent surface B were the same as the lightnesses of surfaces A and D seen directly.

The subject was instructed to base his/her transparency and constancy judgments on the immediate visual impression. Before presenting the experimental stimuli, each subject was questioned about the transparency and constancy of the sample stimuli until the experimenter was satisfied that the criteria for making these judgments were clear to the subject. Several times during the experiment, the experimenter reminded the subject of the criteria for judging a surface to be transparent and for judging whether the lightness seen through the transparent surface was the same as the lightness of a surface seen directly. Twenty paid volunteers served as subjects in the experiment. They were naive concerning the purposes of the experiment. The stimuli were presented in a different random order to each subject.

\section{Results}

The results are presented in Table 1. Rotating a stimulus $180^{\circ}$ interchanges regions $\mathrm{d}$ and $\mathrm{b}$. If stimulus bacd is inverted, it becomes the stimulus dacb. Thus, seeing the bottom surface $D$ as the transparent surface and overlying the top surface $B(D / B)$ with stimulus bacd is the same as seeing the top surface $B$ as the transparent surface and overlying the bottom surface $D(B / D)$ with stimulus dacb. Column 1 shows the 12 pairs of corresponding stimuli. The numbers of transparency judgments were added for corresponding pairs of stimuli. Column 2 shows the total number of times that, for the first stimulus in a pair, the bottom surface $D$ was judged as transparent and, for the second stimulus in a pair, the top surface B was judged as transparent [e.g., (D/B) judgments for stimulus bacd $+(\mathrm{B} / \mathrm{D})$ judgments for stimulus dacb]. These were tabulated as Type 1 judgments. Column 3 shows the total number of times that, for the first stimulus in a pair, the top surface B was seen as transparent and, for the second stimulus in a pair, the bottom surface D was seen as transparent [e.g., (B/D) judgments for stimulus bacd + (D/B) judgments for stimulus dacb]. These were tabulated as Type 2 judgments. The statement of restrictions $i$ through iv assumes that surface $D$ is seen as the transparent surface. If surface $B$ is seen as the transparent surface, then the letters $b$ and $d$ need to be interchanged in restrictions i through iv. For example, restriction iv is violated if surface B is seen as transparent, if the lightness interval $[a, d]$ is contained in the lightness interval $[c, b]$. Table 2 shows that stimuli $b a d c$ and $d a b c$ (stimulus pair 7) satisfy restriction iv for Type 1 judgments. When $D$ is seen as the transparent surface, the lightness interval $[a, b]$ is not contained in the lightness interval $[c, d]$ for stimulus badc, and when B is seen as the transparent surface, the lightness interval $[a, d]$ is not contained in the lightness interval $[c, b]$ for stimulus $d a b c$. Stimuli $b a d c$ and $d a b c$ violate restriction iv for Type 2 judgments. When $B$ is seen as the transparent surface, the lightness interval $[a, d]$ is contained in the lightness interval $[c, b]$ for stimulus $b a d c$, and when $D$ is seen as the transparent surface, the lightness interval $[a, b]$ is contained in the lightness interval $[c, d]$ for stimulus dabc.

The stimuli were divided into four groups. There were eight stimuli in Group I. Table 2 shows that all stimuli in Group I satisfied both the simple and the strong order and magnitude restrictions (i through iv). The transparency judgments for Types 1 and 2 may therefore be considered together. The mean number of transparency judgments was 18.4 (the maximum possible mean value was 20). As in the earlier study of Beck et al. (1984), transparency was readily seen. Color constancy, however, did not always occur, even though restrictions $i$ through iv were satisfied. The mean percentage constancy judgments 
Table 1

Frequency of Transparency Judgments

\begin{tabular}{|c|c|c|c|c|c|c|}
\hline Stimulus & \multirow{2}{*}{$\begin{array}{c}\text { Type } 1 \\
\text { D/B } \\
\text { B/D }\end{array}$} & \multirow{2}{*}{$\begin{array}{c}\text { Type } 2 \\
\text { B/D } \\
\text { D/B }\end{array}$} & \multirow[b]{2}{*}{ Total } & \multirow{2}{*}{$\begin{array}{c}\text { Not } \\
\text { Transparent }\end{array}$} & \multirow{2}{*}{$\begin{array}{l}\text { \% Constancy } \\
\text { (Region c) }\end{array}$} & \multirow{2}{*}{$\begin{array}{c}\% \text { Constancy } \\
\text { (Regions d or b) }\end{array}$} \\
\hline 1st 2nd & & & & & & \\
\hline \multicolumn{7}{|c|}{ Group I } \\
\hline 1. $a b d c a d b c$ & 17 & 14 & 31 & 9 & 32 & 94 \\
\hline 2. bacd dacb & 12 & 26 & 38 & 2 & 95 & 67 \\
\hline 3. bcad dcab & 13 & 25 & 38 & 2 & 95 & 76 \\
\hline 4. $c d b a c b d a$ & 23 & 17 & 40 & 0 & 90 & 95 \\
\hline$M$ & 8.1 & 10.3 & 18.4 & 1.6 & 78 & 83 \\
\hline \multicolumn{7}{|c|}{ Group II } \\
\hline 5. $a d c b a b c d$ & 36 & 1 & 37 & 3 & 100 & 86 \\
\hline 6. $b c d a d c b a$ & 39 & 1 & 40 & 0 & 90 & 100 \\
\hline$M$ & 18.8 & .50 & 19.3 & .75 & 95 & 93 \\
\hline \multicolumn{7}{|c|}{ Group III } \\
\hline 7. $b a d c d a b c$ & 12 & 0 & 12 & 28 & 8 & 92 \\
\hline 8. $c d a b c b a d$ & 21 & 1 & 22 & 18 & 29 & 95 \\
\hline$M$ & 8.3 & .25 & 8.5 & 11.5 & 19 & 94 \\
\hline \multicolumn{7}{|c|}{ Group IV } \\
\hline 9. $a c d b a c b d$ & 12 & 0 & 12 & 28 & 8 & 67 \\
\hline 10. bdac dbac & 1 & 0 & 1 & 39 & 0 & 0 \\
\hline 11. $c a d b c a b d$ & 2 & $\mathbf{0}$ & 2 & 38 & 0 & 0 \\
\hline 12. $b d c a d b c a$ & 0 & $\mathbf{0}$ & $\mathbf{0}$ & 40 & 0 & $\mathbf{0}$ \\
\hline$M$ & 1.9 & $\mathbf{0}$ & 1.9 & 18.1 & 2 & 17 \\
\hline
\end{tabular}

Note-The \% constancy entries are with respect to the stimuli judged transparent. For D/B judgments, D is seen as the transparent surface and $A$ and $B$ as the opaque surfaces. For B/D judgments, $B$ is seen as the transparent surface and $A$ and $D$ as the opaque surfaces.

was $78 \%$ for region $c$ and $83 \%$ for regions b or d. Constancy depends on the pattern of lightnesses in a stimulus. Constancy was least, $32 \%$, when region $\mathrm{c}$ was the lightest area. When region $c$ was not the lightest area in a stimulus, the percentage of transparency judgments exhibiting constancy ranged between $90 \%$ and $95 \%$. A subject was classified as exhibiting constancy if the lightness of the underlying surface seen through area clooked the same as the lightness of the surface seen directly on at least one of the transparency judgments made with a pair of stimuli. For the stimuli $a b d c$ and $a d b c, 7$ subjects (32\% of the transparency judgments) judged region $\mathrm{c}$ to exhibit constancy. For stimuli $c d b a$ and $c b d a, 19$ subjects $(90 \%$ of the transparency judgments) judged region $\mathrm{c}$ to exhibit constancy. Seventeen subjects judged at least one of the stimuli of the pair $a b d c$ and $a d b c$ and also one of the stimuli of the pair $c d b a$ and $c b d a$ as transparent. For these 17 subjects, there were no subjects who exhibited constancy for the pair of stimuli $a b d c$ and $a d b c$ and who failed to exhibit constancy for the pair of stimuli $c d b a$ and $c b d a$. In contrast, 10 of the 17 subjects exhibited constancy for the pair of stimuli $c d b a$ and $c b d a$ and failed to exhibit constancy for the pair of stimuli $a b d c$ and $a d b c$. A McNemar test (Siegel, 1956) of this difference is significant $\left(\chi^{2}=8.1, p<.01\right)$.

There were four stimuli in Group II. Table 2 shows that all Group II stimuli satisfy both the simple and strong magnitude restrictions, ii and iv, for both Type 1 and Type 2 judgments. Type 1 judgments satisfy the simple order restriction i but violate restriction iii. Type 2 judg- ments satisfy restriction iii but violate the simple order restriction $\mathrm{i}$. The mean number of Type 1 judgments was 18.8. The mean number of Type 2 judgments was .50 . The results show that there was a strong preference for satisfying restriction $\mathrm{i}$ over restriction iii when they conflict. The similarity in the mean number of transparency judgments in Groups I and II shows that violation of restriction iii when restriction i was satisfied did not adversely affect perceptual transparency. What apparently is important is the unification of region $c$ with region $d$. For the perception of transparency, continuity of the transparent surface across the boundary of the opaque surfaces is more important than continuity of the underlying opaque surfaces across the boundary of the transparent surface.

There are four stimuli in Group III. Table 2 shows that Type 1 judgments satisfy the simple and strong magnitude restrictions, ii and iv, but violate the simple order restriction, $i$. Type 2 judgments satisfy the simple order restriction, $i$, but violate the simple and strong magnitude restrictions, ii and iv. Type 1 judgments satisfy restriction iii and Type 2 judgments violate restriction iii. The mean number of Type 1 judgments was 8.3 and of Type 2 judgments .25 . The results are similar to those of Beck et al. (1984). Violation of the simple order restriction- $i$ is violated and iii is satisfied-reduced the perception of transparency by about $50 \%$. In contrast, violation of the strong magnitude restriction, iv, strongly suppressed the perception of transparency.

According to the episcotister model, no transparency judgments should occur in Group III. Twelve subjects 
Table 2

Satisfaction (s) and Violation (v) of Restrictions i-iv

\begin{tabular}{|c|c|c|c|c|c|c|c|c|}
\hline \multirow[b]{2}{*}{ Stimulus } & \multicolumn{2}{|c|}{$a>b \stackrel{\mathrm{i}}{\rightarrow} d>c$} & \multicolumn{2}{|c|}{$|a-b| \stackrel{\mathrm{ii}}{>}|d-c|$} & \multicolumn{2}{|c|}{$a>d \stackrel{\text { iii }}{\rightarrow} b>c$} & \multicolumn{2}{|c|}{$\stackrel{\text { iv }}{\in}[c, b] \in[c, d]$} \\
\hline & \multirow{2}{*}{$\begin{array}{c}\text { Type 1 } \\
\text { D/B } \\
\text { B/D } \\
\end{array}$} & \multirow{2}{*}{$\begin{array}{c}\text { Type } 2 \\
\text { B/D } \\
D / B\end{array}$} & \multirow{2}{*}{$\begin{array}{c}\text { Type 1 } \\
\text { D/B } \\
\text { B/D } \\
\end{array}$} & \multirow{2}{*}{$\begin{array}{c}\text { Type } 2 \\
\text { B/D } \\
\text { D/B }\end{array}$} & \multirow{2}{*}{$\begin{array}{c}\text { Type } 1 \\
\text { D/B } \\
\text { B/D }\end{array}$} & \multirow{2}{*}{$\begin{array}{c}\text { Type } 2 \\
\text { B/D } \\
\text { D/B } \\
\end{array}$} & \multirow{2}{*}{$\begin{array}{c}\text { Type 1 } \\
\text { D/B } \\
\text { B/D } \\
\end{array}$} & \multirow{2}{*}{$\begin{array}{c}\text { Type } 2 \\
\text { B/D } \\
\text { D/B }\end{array}$} \\
\hline 1st 2nd & & & & & & & & \\
\hline \multicolumn{9}{|c|}{ Group I } \\
\hline \multirow{4}{*}{$\begin{array}{l}\text { 1. } a b d c a d b c \\
\text { 2. } b a c d d a c b \\
\text { 3. } b c a d d c a b \\
\text { 4. } c d b a c b d a\end{array}$} & $\mathbf{s}$ & s & $\mathbf{s}$ & $\mathbf{s}$ & s & $\mathbf{s}$ & $\mathbf{s}$ & $\mathbf{s}$ \\
\hline & s & $\mathbf{s}$ & $\mathbf{s}$ & $\mathbf{s}$ & $\mathbf{s}$ & $\mathbf{s}$ & $\mathbf{s}$ & s \\
\hline & $\mathbf{s}$ & $\mathbf{s}$ & $\mathbf{s}$ & s & s & s & $\mathbf{s}$ & s \\
\hline & $\mathbf{s}$ & $\mathbf{s}$ & $\mathbf{s}$ & $\mathbf{s}$ & $\mathbf{s}$ & s & $\mathbf{s}$ & $\mathbf{s}$ \\
\hline \multicolumn{9}{|c|}{ Group II } \\
\hline \multirow{2}{*}{$\begin{array}{l}\text { 5. adcb abcd } \\
\text { 6. } b c d a d c b a\end{array}$} & $\mathbf{s}$ & $\mathbf{v}$ & s & $\mathbf{s}$ & $\mathbf{v}$ & s & $\mathbf{s}$ & $\mathbf{s}$ \\
\hline & $\mathbf{s}$ & $\mathbf{v}$ & $\mathbf{s}$ & s & $\mathbf{v}$ & $\mathbf{s}$ & $\mathbf{s}$ & $\mathbf{s}$ \\
\hline \multicolumn{9}{|c|}{ Group III } \\
\hline \multirow{2}{*}{$\begin{array}{l}\text { 7. badc dabc } \\
\text { 8. } c d a b c b a d\end{array}$} & $\mathbf{v}$ & $\mathbf{s}$ & $\mathbf{s}$ & $\mathrm{v}$ & $\mathbf{s}$ & $\mathbf{v}$ & $\mathbf{s}$ & $\mathbf{v}$ \\
\hline & $\mathbf{v}$ & $\mathbf{s}$ & $\mathbf{s}$ & $\mathbf{v}$ & $\mathbf{s}$ & $\mathbf{v}$ & $\mathbf{s}$ & $\mathbf{v}$ \\
\hline \multicolumn{9}{|c|}{ Group IV } \\
\hline \multirow{4}{*}{$\begin{array}{l}\text { 9. } a c d b \text { acbd } \\
\text { 10. bdac dbac } \\
\text { 11. cadb cabd } \\
\text { 12. bdca dbca }\end{array}$} & $\mathbf{v}$ & $\mathbf{v}$ & $\mathbf{s}$ & $\mathbf{s}$ & $\mathbf{v}$ & $v$ & $\mathbf{s}$ & $\mathbf{s}$ \\
\hline & $\mathbf{v}$ & $\mathbf{v}$ & $\mathbf{s}$ & $v$ & $v$ & $v$ & $\mathbf{s}$ & $\mathbf{v}$ \\
\hline & $v$ & $\mathbf{v}$ & $\mathbf{s}$ & $v$ & $\mathbf{v}$ & $\mathbf{v}$ & $\mathbf{s}$ & $\mathbf{v}$ \\
\hline & $v$ & $\mathbf{v}$ & s & $\mathbf{s}$ & $v$ & $\mathbf{v}$ & $\mathbf{s}$ & $\mathbf{s}$ \\
\hline
\end{tabular}

Note-For D/B judgments, $D$ is seen as the transparent surface and $A$ and $B$ as the opaque surfaces. For $B / D$ judgments, $\mathrm{B}$ is seen as the transparent surface and $\mathrm{A}$ and $\mathrm{D}$ as the opaque surfaces. For $\mathrm{B} / \mathrm{D}$ judgments, the letters $b$ and $d$ need to be interchanged in restrictions $i$ through iv.

(60\%) judged either stimulus badc or stimulus dabc as transparent, and $14(70 \%)$ judged either stimulus $c d a b$ or stimulus cbad (or both) as transparent. A $99 \%$ confidence interval for stimuli badc and dabc is $.30 \leq p \leq$ .85 and for stimuli $c d a b$ and $c b a d$ is $.39 \leq p \leq .92$. The value 0 falls outside both confidence intervals. The results, therefore, are not consistent with the episcotister model.

It is of interest that violation of the simple order restriction i in Group III reduced color constancy. Fewer Group III subjects than Group I subjects reported that the lightness seen through area c looked the same as the lightness of the underlying surface. As above, a subject was classified as showing constancy if he/she judged area $c$ to exhibit constancy on at least one of the transparency judgments made with a pair of stimuli. In Group III when region $\mathrm{c}$ was the lightest area in a stimulus (stimuli badc and $d a b c)$, constancy occurred for only 1 subject $(8 \%$ of the transparency judgments), and when $\mathrm{c}$ was the darkest area in a stimulus (stimuli $c d a b$ and $c b a d$ ), constancy $o c-$ curred for 5 subjects (29\% of the transparency judgments). As mentioned above, comparable scores for Group I when region c was the lightest (stimuli $a b d c$ and $a d b c$ ) and darkest (stimuli $c d b a$ and $c b d a$ ) area in a stimulus were 7 subjects and 19 subjects, respectively. Eleven subjects judged at least one of the stimuli of the pair $a b d c$ and $a d b c$ and one of the stimuli of the pair $b a d c$ and $d a b c$ as transparent. For these 11 subjects, there were no subjects who exhibited constancy for the pair of stimuli badc and $d a b c$ and who failed to exhibit constancy for the pair of stimuli $a b d c$ and $a d b c$. In contrast, 5 subjects exhibited constancy for the pair of stimuli $a b d c$ and $a d b c$ and failed to exhibit constancy for the pair of stimuli badc and dabc. A binomial test of the difference is not significant $(p>.05)$. Thirteen subjects judged at least one of the stimuli of the pair $c d b a$ and $c b d a$ and one of the stimuli of the pair $c d a b$ and $c b a d$ as transparent. For these 13 subjects, there were no subjects who exhibited constancy for the pair of stimuli $c d a b$ and $c b a d$ and who failed to exhibit constancy for the pair of stimuli $c d b a$ and $c b d a$. In contrast, 8 of the 13 subjects exhibited constancy for the pair of stimuli $c d b a$ and $c b d a$ and failed to exhibit constancy for the pair of stimuli $c d a b$ and $c b a d$. A binomial test of the difference is significant $(p<.01)$.

There are eight stimuli in Group IV. Table 2 shows that all stimuli violate the simple and strong order restrictions, i and iii, for both Type 1 and Type 2 judgments. They differ in their satisfaction of the simple and strong magnitude restrictions, ii and iv. Stimuli 9 and 12 satisfy them for both Type 1 and Type 2 judgments. Stimuli 10 and 11 satisfy the simple and strong magnitude restrictions for Type 1 judgments, but not for Type 2 judgments. The mean number of transparency judgments was 1.9. When the order restriction was strongly violated, the perception of transparency did not occur except for stimuli $a c d b$ and $a c b d$. Ten subjects (50\%) judged either stimulus $a c d b$ or stimulus acbd as transparent. A $99 \%$ confidence interval is $.27 \leq p \leq .73$. According to both the episcotister model and the figural organizational model, no 
transparency judgments should occur in Group IV. The value 0 falls outside this confidence interval. We comment on this in the Discussion.

\section{Discussion}

The visual system is adaptive. Our perceptions of transparency have to be in accord with reality in important respects. This does not mean, however, that the visual system uses luminances to compute indices of transmittance and reflectance analogous to what an optical engineer might do in describing a physical instance of transparency. One may take the view that the Metelli derivation is an algorithm for predicting what will be seen and is not a description of visual processing. The visual system does not split the luminance in the overlapping region into two components-the lightness of the surface seen through the transparent surface and the lightness of the transparent surface. The hypothesis proposed is that in the absence of depth cues, such as stereopsis and motion parallax, the perception of transparency is determined by organizational processes. Organizational processes determine whether area $c$ is seen as separate and not transparent or whether area $c$ is joined to area $d$ and transparency is seen. Factors affecting this structuring are specific cues (e.g., the lightness value of the overlapping region; see below) and the similarity relations based on the direction of lightness value changes and the relative similarities of the lightness values in a stimulus. The perception of transparency may be accompanied by an encoding of the lightness value of the overlapping region as the lightness of an underlying opaque surface modified by the transmittance of the overlying transparent layer. This, however, is not a necessary condition.

Figural and lightness cues cooperate in yielding the perception of transparency. An x-junction suggests overlying surfaces and transparency. Lightness values of the overlapping region that satisfy the order and magnitude restrictions are consistent with a perception of transparency and facilitate seeing transparency. Some subjects tended not to see transparency unless the perceived lightness of the surface seen through the transparent layer was seen the same as the perceived lightness of the surface seen directly. Other subjects were apparently not disturbed by the fact that the perceived lightness of the underlying surface in the transparent region did not appear correct and still reported a stimulus as transparent. Constancy in the experiment occurred when the lightnesses of the underlying surfaces seen through the transparent layer were seen to be the same as the lightnesses of the surfaces not seen through the transparent layer. None of the subjects exhibited constancy for all stimuli; that is, every subject reported the perception of transparency in at least one instance in which the lightness of the underlying surface seen through the transparent layer was not seen to be the same as the lightness of the surface seen directly. There were 86 judgments of transparency for which either surface A or surface B (for Type 1 judgments) or either surface A or surface D (for Type 2 judgments) were judged as not exhibiting constancy. Seven subjects ( 10 judgments) judged a stimulus transparent when both surfaces $A$ and B (or D) failed to exhibit constancy. The results clearly show that transparency can occur without color constancy. What is suggested is that the perception of an inappropriate lightness of the underlying surface overrode the transparency suggested by the occluding contours for some subjects but not for others.

Unlike in Beck et al.'s (1984) study, transparency judgments in the present study occurred with stimuli $a c d b$ and $a c b d$. The occurrence of transparency judgments with these stimuli shows that a strong violation of the order restrictions $i$ and iii does not completely preclude the perception of transparency. Informal observations indicate that the perception of transparency for some subjects was not accompanied by a perception that the lightness of the underlying surface is incorrect. Subjects, however, can be made sensitive to perceptions of lightness that are inconsistent with the perception of transparency. Exploratory experiments in which the instructions called attention to the incorrectness of the perceived lightnesses of the underlying surfaces in the transparent region reduced the number of transparency judgments in Groups III and IV. It is of interest that transparency judgments occurred only with stimuli $a c d b$ and $a c b d$ and not for other stimuli in Group IV. The reason for this is not completely evident. One possibility is that the perception of transparency is favored both when $c$ is darker than $b$ or $d$ and when $c$ is close in lightness to $b$ and $d$. These two conditions hold only for stimuli $a c d b$ and $a c b d$.

Metelli et al. (1985) accepted Beck et al.'s (1984) findings that the perception of transparency is determined by lightness values and not by the physical variables of luminance or reflectance. Metelli et al. also accepted the findings that the restrictions $\mathrm{i}^{*}$ and $\mathrm{ii}^{*}$, derived from the equation for the reflectance of the episcotister blades, do not affect the perception of transparency. They argued, however, that we are mistaken in believing that figural organization is a primary factor in the perception of transparency. We believe that the role of figural organization becomes evident when the distinction between simple and strong violations of the order and magnitude restrictions is recognized. For example, Metelli et al. reported results in which figural organization cues resulting from pattern movement failed to produce transparency when restrictions $i$ and ii were violated. The stimuli for which motion did not give rise to the perception of transparency are those that we have described as strong violations. For two stimuli (stimuli 19 and 20 in Table 4 of Metelli et al., $1985)$ the lightness interval $[a, b]$ was contained within the lightness interval $[c, d]$-that is, the stimuli violated the strong magnitude restriction (iv)-and for six stimuli (stimuli 11-16 in their Table 4) the pattern of lightnesses gave a crisscross pattern-that is, the stimuli strongly violated the strong order restriction ( $\mathrm{i}$ and iii). They also presented examples of figures in which transparency is reported when there is no superposition or $\mathrm{x}$-junction. Their subjects, however, were not naive. It is possible to see transparency under these conditions, but in our view the perception of transparency is not stimulus determined. 
It depends on a subject's adopting an attitude that favors the perception of transparency. Our research is concerned with the perception of transparency when it is stimulus controlled. We do not believe naive subjects would generally see transparency when there are no $x$-junctions or superpositions of figures.

Metelli et al. (1985) also found a significant number of transparency judgments for stimuli $b a d c$ and $c d a b$. These are stimuli that violate the simple order restriction (i), but not the strong order restriction (i and iii). Their subjects, however, reported these stimuli as instances of partial transparency, which refers to the perception of transparency in which a surface is transparent in part and opaque in part. We explicitly instructed our subjects to report a surface as not transparent if they saw a part of the surface as opaque. Nonetheless, subjects reported stimuli badc and $c d a b$ as transparent. The conflicting results are explained if for sophisticated subjects the perception of transparency tends to be inhibited when the perceived lightness of the underlying surface seen through the transparent layer is different from the perceived lightness of the surface seen directly.

The eight stimuli in Group I satisfy restrictions $\mathrm{i}$ through iv when surface $D$ was seen as lying over surface $B$ or when surface $D$ was seen as lying under surface B. Auxiliary rules are therefore necessary to predict whether $D$ is seen as the transparent surface or whether B is seen as the transparent surface. Beck et al. (1984) suggested that region $c$ is joined to regions $b$ or $d$, depending on which it differs from least in lightness. In that study, Beck et al. reported results supporting this principle. However, this rule was violated in the present study. The majority of subjects saw B as the overlying transparent surface for stimulus bacd and D as the overlying transparent surface for stimulus dacb. One difference between the present study and the earlier study of Beck et al. (1984) is that region $d$ or region $b$ in that study was never the darkest area of a stimulus. The results suggest that there is also a bias to see the darkest area of a stimulus as transparent. The source of the bias may be that when looking through a filter, the darkest area in the scene is normally seen through the filter. Another instance in which specific lightness values influenced the perception of transparency was reported by Kozaki (1974). He found that increasing the lightness of the background increases the likelihood to see the white rather than the black of two intersecting rectangles as transparent. The perception of transparency is affected by a variety of factors, in addition to the restrictions $i$ through iv, that have their origins in an individual's experience with transparent surfaces.

\section{REFERENCES}

BECK, J. (1986a). The perception of transparency: A reply to Brill. Computer Vision, Graphics, \& Image Processing, 35, 272-273.

BECx, J. (1986b). Perception of transparency in man and machine. In A. Rosenfeld (Ed.), Human and machine vision II (pp. 1-12). New York: Acadernic Press.

Beck, J., Prazdny, K., \& Ivry, R. (1984). The perception of transparency with achromatic colors. Perception \& Psychophysics, 35 , 407-422.

BriLL, M. H. (1986). Perception of transparency in man and machine: A comment on Beck. Computer Vision, Graphics, \& Image Processing, 35, 270-271.

Fuchs, W. (1923). Experimentelle Untersuchungen uber das simultane Hintereinandersehen auf der selben Sehrichtung. Zeitschrifi fur Psychologie, 91, 145-235.

JuDD, D. B., WYSZECKI, G. (1963). Color in business, science, and industry. New York: Wiley.

KozAKI, T. (1974). The role of background lightmess in the apparent iransparency (Report No. 7). Yokohama, Japan: Keio University, Psychological Laboratory.

METELL, F. (1974). Achromatic color conditions in the perception of transparency. In R. B. MacLeod \& H. L. Pick (Eds.), Perception: Essays in honor of J. J. Gibson (pp. 95-116). Ithaca, NY: Cornell University Press.

METELLI, F. (1985). Stimulation and perception of transparency. Psychological Research, 47, 185-202.

Metelli, F., Da Pos, O., Cavedon, A. (1985). Balanced and unbalanced, complete and partial transparency. Perception \& Psychophysics, 38, 354-366.

METZGER, W. (1955). Uber Durchsichtigkeits-Erscheinungen. Revista di Psicologia, 49, 187-189.

SIEGEL, S. (1956). Nonparametric statistics in the behavioral sciences. New York: McGraw-Hill.

\section{NOTES}

1. The derivation in Beck et al. (1984) is for a filter lying on a surface in which the illumination is coming from in front. Transparency can occur in a variety of physical ways, and the restrictions $i, i^{*}$, ii, and $\mathrm{ii}^{*}$ hold for other conditions as well. The relevant restrictions for the perception of transparency are $i$ and ii. The generality of restrictions $i$ and ii becomes evident if they are translated into words. Restriction $i$ says that a homogeneous transparent surface cannot change the order of intensities reflected from surfaces. If the intensity of surface $A$ is greater than that of surface $B$, then viewing surfaces $A$ and $B$ through a transparent surface cannot make the intensity of surface B greater than that of surface A. Restriction ii says that a transparent surface reduces but cannot increase the intensity difference between two surfaces.

2. The term lighmess here refers to the Munsell values matched to the regions in a stimulus when the regions are seen as coplanar and transparency is not seen, that is, the lightnesses of the stimulus regions on the picture surface. The term lightness is also used to refer to the perceived lightness of the underlying surface seen through the transparent surface in pictorial space. The context generally makes clear which of the two senses is intended. When confusion is possible, lightness value will be used interchangeably with the term lightness. Lightness value always refers to the lightness of a stimulus region on the picture surface.

(Manuscript received August 19, 1987; revision accepted for publication June 8,1988 .) 